\title{
DESENSITIZING METHOD FOR MEASUREMENT OF THIRD-ORDER INTERMODULATION DISTORTION IN CMOS-MEMS MICROMECHANICAL RESONATORS
}

\author{
Chiung-Cheng Lo ${ }^{1}$, and G. K. Fedder ${ }^{1,2}$ \\ ${ }^{1}$ Department of Electrical and Computer Engineering and ${ }^{2}$ The Robotics Institute \\ Carnegie Mellon University, Pittsburgh, PA, 15213 \\ Email: Chiungcl@ece.cmu.edu Tel: 412-268-8194 Fax: 412-268-4594
}

\begin{abstract}
A new desensitization method to characterize linearity of micromechanical resonator filters is introduced. The practical measuring range is larger than either the conventional $1 \mathrm{~dB}$ compression test or the two-tone test for RF frequencies. An analytic expression for a new $1 \mathrm{~dB}$ desensitizing point $\left(\mathrm{DP}_{1 \mathrm{~dB}}\right)$ metric is directly related to the $1 \mathrm{~dB}$ compression point $\left(\mathrm{CP}_{1 \mathrm{~dB}}\right)$. This method is verified on a CMOS-MEMS square-frame resonator (SFR) with resonant frequency of $5.849 \mathrm{MHz}$, quality factor of 1268 and insertion gain of $-31.8 \mathrm{~dB}$ and $-41.7 \mathrm{~dB}$ for input polarization voltage of $10 \mathrm{~V}$ and $30 \mathrm{~V}$, respectively. The measured $\mathrm{DP}_{1 \mathrm{~dB}}$ is $26.7 \mathrm{Vac}$ corresponding to a $\mathrm{CP}_{1 \mathrm{~dB}}$ of $32 \mathrm{dBm}$.
\end{abstract}

\section{INTRODUCTION}

Recent progress of microresonators as passive components in wireless communication applications achieves high quality factor (Q) and high operational frequency. For example, hollow-disk ring resonators have been demonstrated with $\mathrm{Q}>60,000$ at $24 \mathrm{MHz}$ and $\mathrm{Q}>14,000$ at $1.2 \mathrm{GHz}$ [1]. By mechanical or electrostatic coupling, MEMS resonators yield filtering and mixing functions with miniature size [2-4]. However, in order to fulfill strict wireless communication specifications, such as the minimum total thirdorder input intercept point (IIP3) of $-18 \mathrm{dBm}$ in the signal path for the European GSM standard [5], the measurement and the modeling of the nonlinear effects of micro resonators is critical.

Nonlinear effects of microresonators are presented, and two types of linearity measurement are discussed in part 1 . In part 2, a new desensitization method is introduced, the measurement set-up is illustrated, and the mechanism of this method is examined. A series of experiments are conducted and the validation of this measurement method is analyzed in part 3 .

(A)
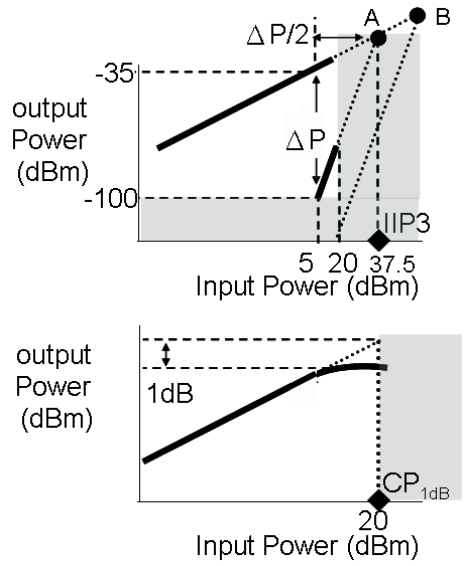

Figure 1. Example of measured range for (A) a two-tone test and (B) a $1 \mathrm{~dB}$ compression test. Shaded areas are inaccessible for measurement.

\section{NONLINEAR MICRORESONATORS}

Nonlinearity of the communication systems, especially the third-order intermodulation distortion, is critical to quantify because it usually defines the upper bound of the power handling ability. Nonlinearity occurs when out-of-band tones are modulated and results in generation of in-band components. When the input signal, $V_{i n}$, consists of a desired signal, $V_{i} \cos \omega_{i} t$, and two out-ofband interference tones, $V_{i m-1} \cos \omega_{i m-1} t$ and $V_{i m-2} \cos \omega_{i m-2} t$, the output components after transferring through a nonlinear system are given by [6]

$$
\begin{gathered}
\text { (a) (b) (c) } \\
V_{o}=\left(a_{1} V_{i}+\frac{3 a_{3} V_{i}^{3}}{4}+\frac{3 a_{3} V_{i} V_{i m-1}^{2}}{2}+\frac{3 a_{3} V_{i} V_{i m-2}^{2}}{2}\right) \cos \omega_{i} t \\
+\frac{3 a_{3} V_{i m-1}^{2} V_{i m-2}}{4} \cos \left(\left(2 \omega_{i m-1}-\omega_{i m-2}\right) t+\frac{3 a_{3} V_{i m-1} V_{i m-2}^{2}}{4} \cos \left(\left(2 \omega_{i m-2}-\omega_{i m-1}\right) t+\ldots\right.\right.
\end{gathered}
$$

where component (a) is the fundamental tone and (e) and (f) are the intermodulation tone (IM). The nonlinear system in (1) is modeled as

$$
V_{o}=a_{1} V_{\text {in }}+a_{2} V_{\text {in }}^{2}+a_{3} V_{\text {in }}^{3} \cdots
$$

In order to quantify this phenomenon, the two-tone test has been applied by other groups [7-8]. This method measures the effect of component (e) and (f) in (1). The frequencies of the two tones are selected to maximize the IM tone, given by

$$
2 \omega_{i m-1}-\omega_{i m-2}=\omega_{r} \quad \text { or } \quad 2 \omega_{i m-2}-\omega_{i m-1}=\omega_{r}
$$

where $\omega_{\mathrm{r}}$ is the resonant frequency. By plotting the output power versus input power for the fundamental tone and IM tone, the intercept of the extrapolation of these two lines is IIP3, shown in Figure 1(A). In addition, IIP3 is given by [6]

$$
I I P 3_{d B m}=\frac{\Delta P_{d B}}{2}+P_{i n, d B m}
$$

Where $\mathrm{P}_{\text {in }}$ is the input power and $\Delta \mathrm{P}$ is the power difference between fundamental and IM tone. However, the mechanical filtering characteristic suppresses off-resonance output signals to some degree, which degrades the accuracy of extrapolating IIP3. This problem is magnified in the case of high quality factor resonators. The measurement range of this method is a function of dynamic range of measurement instruments, testing range, and the insertion loss of the measured device. For example, in the case that the maximum output power of signal generator is $20 \mathrm{dBm}$, the noise floor of spectrum analyzer is $-100 \mathrm{dBm}$, the insertion loss of the measured device is $40 \mathrm{~dB}$, and the testing range is 

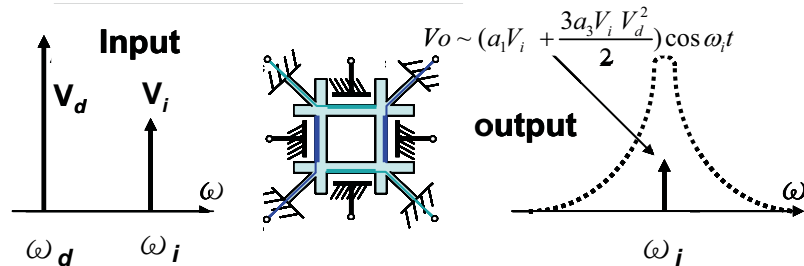

Figure 2. Desensitization test of intermodulation in a nonlinear resonator

$15 \mathrm{~dB}$, the intercept is point (A) shown in Figure 1(A), and the corresponding IIP3 is $37.5 \mathrm{dBm}$, which is approximately the upper bound of measurement range in the two-tone test. For a highly linear device with IIP3 in point (B), the IM tone is below the noise floor and IIP3 is not measurable.

Another method to determine the linearity is to measure the $\mathrm{CP}_{1 \mathrm{~dB}}$, which measures the effect of component (b) in (1). Nevertheless, if the nonlinear component is too weak relative to the component (a), then a high amplitude signal source is needed to cause measurable nonlinearity. This large input signal requirement limits the measurement range of this method. Given a maximum RF source power of around $20 \mathrm{dBm}$, the nonlinearity can be measured on microresonators with $\mathrm{CP}_{1 \mathrm{~dB}}$ less than $20 \mathrm{dBm}$, as shown in Figure 1(B). The corresponding IIP3 is about $29.6 \mathrm{dBm}$, which is given by [6]

$$
I I P 3_{d B m}=C P_{1 d B}+9.6
$$

This relation is an approximation based on (2). Because of the effect of mechanical filtering, (5) might not hold for every situation, and a detailed IIP3 model for the tested device is needed.

\section{DESENSITIZATION METHOD}

As shown in Figure 2, this work introduces a desensitization method to measure the linearity of HF CMOS-MEMS squareframe resonator that consists of four free-free beams connected at the nodal points of the first flexural resonant mode [9]. While introducing a low-frequency strong desensitizing tone at $\omega_{\mathrm{d}}$ with high-frequency desired signal within the filter passband at $\omega_{\mathrm{i}}$, the insertion gain will drop due to the third-order intermodulation effect, which is similar to component (c) or (d) in (1). The advantages of this method are that: first, it measures only the resonant output signal, which is not affected by the off-resonance suppression. Second, due to lower frequency, it is easy to obtain a large-magnitude signal source for the desensitizing tone. This extends the measuring range for testing highly linear devices. Third, fewer instruments are required than in the two-tone test. This method needs only a network analyzer and one lowfrequency signal source, compared to two high-frequency signal sources and a spectrum analyzer for the two-tone test.

The schematic of the measurement setup is shown in Figure 3. The input signal, $V_{i} \cos \omega_{i} t$, the input polarization voltage, $V_{p}$, and the desensitizing tone, $V_{d} \cos \omega_{d} t$, are applied across the input electrodes. Fundamental force and nonlinear forces are given by

$$
F=\frac{\partial C_{i}}{\partial x}\left(V_{i} \cos \omega_{i} t-V_{d} \cos \omega_{d} t-V_{p}\right)^{2}+\frac{\partial C_{o}}{\partial x}\left(V_{p o}\right)^{2}
$$

where $C_{i}$ and $C_{o}$ are input and output capacitance, $x$ is the mechanical displacement and $V_{p o}$ is the output polarization voltage. With the assumption that mechanical displacement is much smaller than the electrode gap, (6) can be expanded into a Taylor series. With small electrode gaps, mechanical spring nonlinearities are negligible compared to the capacitive nonlinear effect [7]. The mechanical displacement is solved from the equation of motion with perturbation techniques [10]. The output voltage components at resonant frequency are

$$
\begin{aligned}
& V_{O, f i}=\left[\left(i \frac{4 C^{2} Q V_{p} V_{p o}}{g^{2} K}-i \frac{32 C^{4} Q^{3} V_{d}^{2} V_{p}^{3} V_{p o}}{g^{6} K^{3}}+\frac{8 C^{3} Q^{2} V_{d}^{2} V_{p} V_{p o}}{g^{4} K^{2}}\right) V_{i}\right. \\
& \left.-i \frac{72 C^{4} Q^{3} V_{p}^{3} V_{p o}}{g^{6} K^{3}} V_{i}^{3}+\ldots\right] \omega_{i} Z
\end{aligned}
$$

where $C_{i}$ and $C_{o}$ are replaced by $C$ with the assumption of negligible mismatch, $Q$ is the quality factor, $g$ is the electrode gap, $K$ is mechanical stiffness, $Z$ is the transimpedance of the on-chip amplifier. The second and third terms of (7) are the first two substantial nonlinear output components for the tested device with the parameters listed in the Table 1 . If the second term is the largest nonlinear component, the magnitude of output the voltage will decrease with increasing desensitizing voltage. However, if the third term is largest, the magnitude of the output voltage will increase with increasing desensitizing voltage.

\section{EXPERIMENTAL RESULTS}

The SEM picture of the tested resonator is shown in Figure 4. To model this resonator, results from a series of tests of sweeping input polarization voltage are shown in Figure 5. The resonant

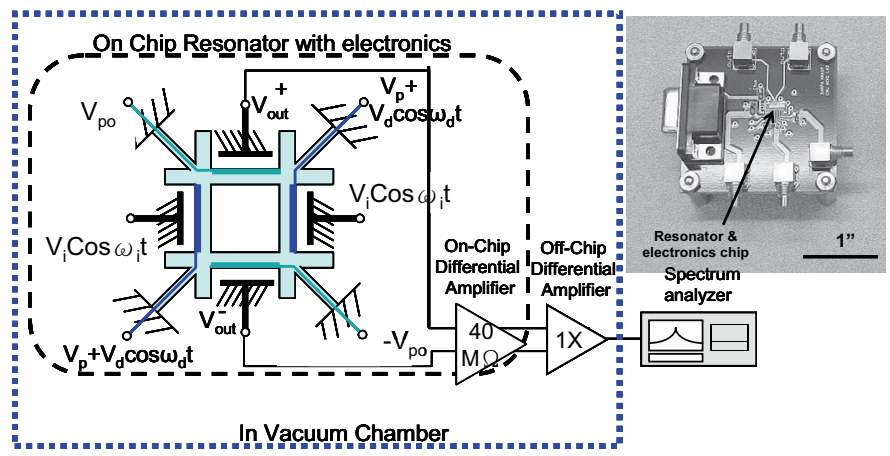

Figure 3. The linearity measurement set-up for the desensitizing method and the perspective view of a resonator chip on a customdesigned test board.

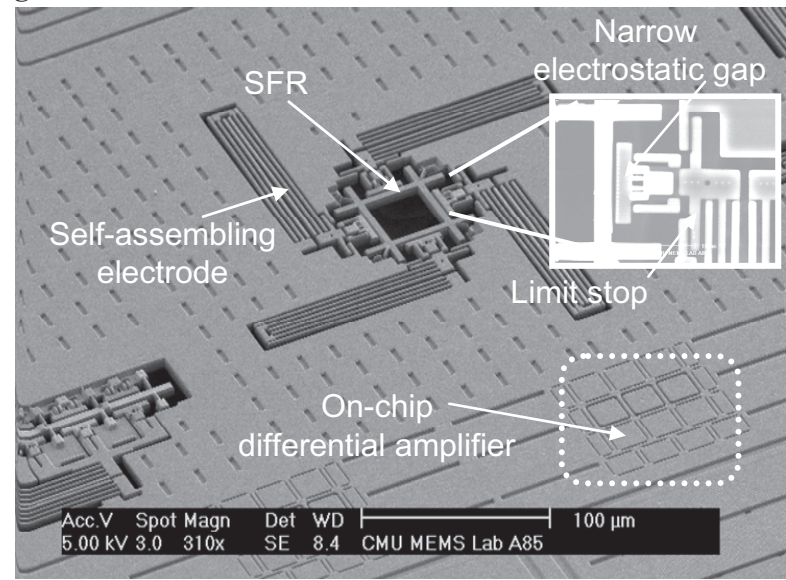

Figure 4. The SEM picture of a SFR with self-assembling electrodes to provide small electrode gaps. [9] 

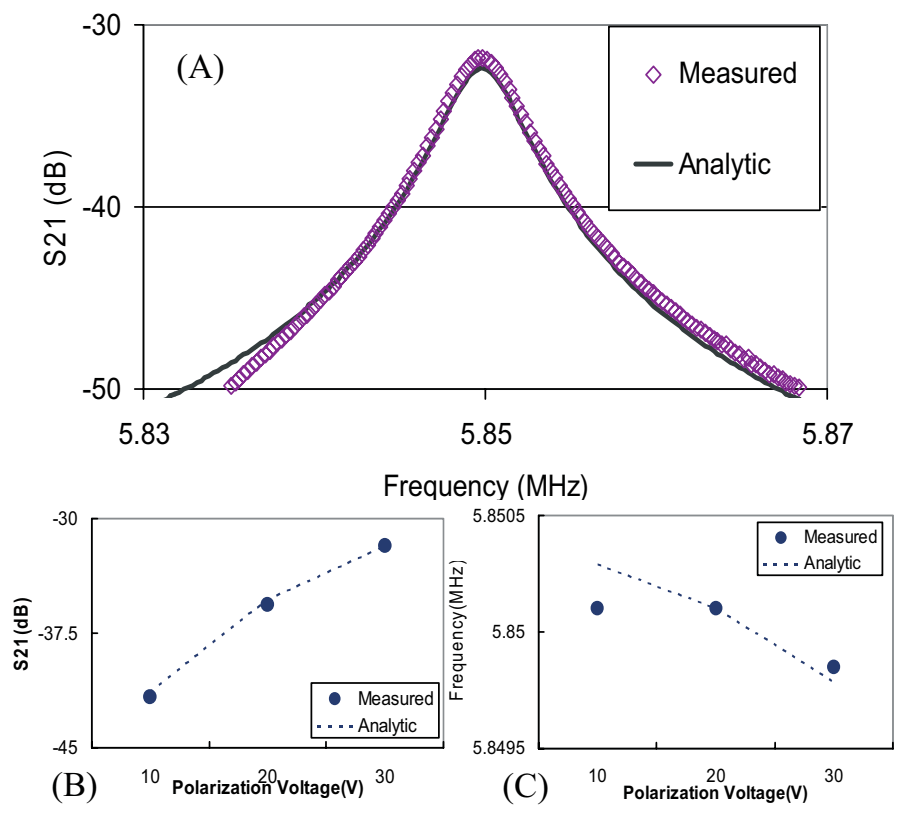

Figure 5. (A) Measured response and analytic solution of SFR with $V_{p}$ of $30 \mathrm{~V}, 20 \mathrm{mTorr}$ of pressure, and $V_{\text {in }}$ of $10 \mathrm{dBm}$ for $1^{\text {st }}$ flexural resonant mode. The parasitic effect is measured and subtracted. (B) and $(C)$ are the extracted measured result and analytic solution of maximum $S_{21}$ and resonant frequency versus $V_{p}$, respectively

frequency is $5.849 \mathrm{MHz}$, the quality factor is 1268 , and the insertion gain is $-31.8 \mathrm{~dB}$ and $-41.7 \mathrm{~dB}$ for $V_{p}$ of $10 \mathrm{~V}$ and $30 \mathrm{~V}$, respectively. Figure 5(A) shows the measured frequency response with the analytic solution, which is calculated from the features listed in the Table 1. The effect of input polarization voltage in the $\mathrm{S}_{21}$ and resonant frequency is measured and calculated, shown in Figure 5(B) and (C). The measurement result is consistent with analytic model, showing that increasing the $V_{p}$ will increase $\mathrm{S}_{21}$ and lower the resonant frequency. The discrepancy at low $V_{p}$ in the Figure 5(C) might result from the measurement error with small magnitude signal and insufficient measurement resolution.

Frequency responses with different desensitizing voltages are measured, shown in Figure 6. The measured maximum $\mathrm{S}_{21}$ values are extracted and plotted with the analytic solution in Figure 7. The analytic solution is modeled by (7) with the parameters listed in the Table 1 where the second component is the dominant nonlinear effect and causes $S_{21}$ drop while increasing desensitizing voltage. With 20 Vac of desensitizing voltage, the $\mathrm{S}_{21}$ drops to $0.55 \mathrm{~dB}$; this is slightly larger than analytic result of $0.49 \mathrm{~dB}$. This measurement validates the features of tested device listed in the Table 1 and the nonlinear model based on (7).

In order to compare the linearity performance of different devices, the $\mathrm{DP}_{1 \mathrm{~dB}}$ is defined as the magnitude of desensitizing tone to cause $1 \mathrm{~dB}$ drop in $\mathrm{S}_{21}$. Neglecting other nonlinear components except the dominant second term in (7), the $\mathrm{DP}_{1 \mathrm{~dB}}$ is

$$
\mathrm{DP}_{1 \mathrm{~dB}}=0.116 \frac{\mathrm{g}^{2} \mathrm{~K}}{C V_{p} Q}
$$

For the tested devices, the $\mathrm{DP}_{1 \mathrm{~dB}}$ is 26.7 Vac. The $\mathrm{CP}_{1 \mathrm{~dB}}$ is calculated to be $32 \mathrm{dBm}$, caused by the forth term in (7), related to $\mathrm{DP}_{1 \mathrm{~dB}}$ by

$$
\mathrm{CP}_{1 \mathrm{~dB}}=20 \log \left(\frac{\mathrm{DP}}{0.16}\right)-12.19
$$

This superior linearity is due to a medium size electrical gap of $0.84 \mu \mathrm{m}$, compared to the results in other work [7-8]. A plot of $\mathrm{DP}_{1 \mathrm{~dB}}, \mathrm{CP}_{1 \mathrm{~dB}}$ and $\mathrm{S}_{21}$ versus electrode gap size is shown in Figure 8, which shows a trade-off between linearity and insertion gain in reducing electrode gap size.

The desensitization method is eventually limited by the nonlinear effects in amplifiers and the network analyzer, and by electrical breakdown on the tested device at high values of $V_{d}$. While there are strong tones along with fundamental tone in the input of amplifiers and network analyzer, the nonlinear effect of those stages blocks the fundamental tone, the mechanism of which is similar to the phenomena shown in (1). Output spectrums with

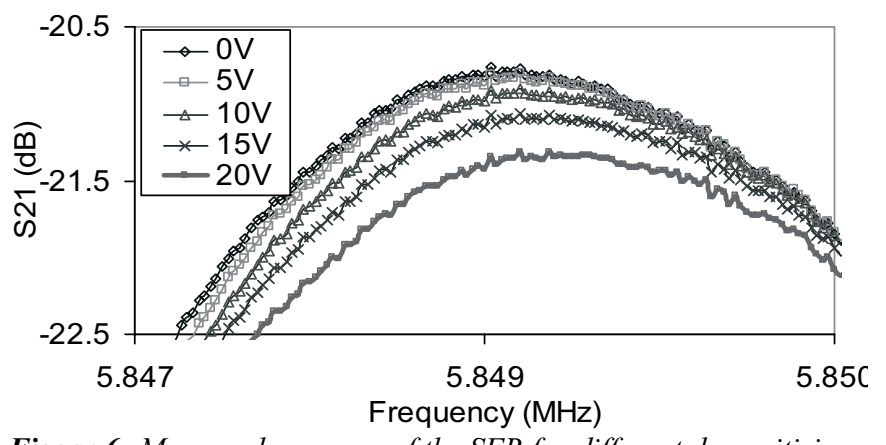

Figure 6. Measured response of the SFR for different desensitizing voltages, $V_{d}$, with $f_{d}=1 \mathrm{kHz}, V_{p}=42.5 \mathrm{~V}, \quad V_{p o}=45 \mathrm{~V}$, pressure $=11 \mathrm{mTorr}$, and $V_{\text {in }}=10 \mathrm{dBm}$.

Table 1. Features of tested microresonators

\begin{tabular}{|c|c|c|}
\hline & Measured & Analytic \\
\hline Beam Length $(\mu \mathrm{m})$ & 64.7 & - \\
\hline Beam Width $(\mu \mathrm{m})$ & 4.6 & - \\
\hline Beam Thickness $(\mu \mathrm{m})$ & - & 5.0 \\
\hline Input/Output Capacitor Width $(\mu \mathrm{m})$ & - & 20 \\
\hline Input/Output Capacitor Gap, $\mathrm{g}(\mu \mathrm{m})$ & - & 0.84 \\
\hline $\begin{array}{c}\text { Output Polarization Voltage, } \mathrm{V}_{\mathrm{po}}(\mathrm{V}) \\
\text { (for tests in the Figure 5) }\end{array}$ & $20 /-17.4$ & 18.7 \\
\hline $\begin{array}{c}\text { Input/output Polarization Voltage, } \mathrm{V}_{\mathrm{p}}(\mathrm{V}) \\
\text { (for tests in the Figure 6) }\end{array}$ & $42.5 / 45$ & $45.5 / 47$ \\
\hline Mechanical Stiffness, $\mathrm{K}(\mathrm{N} / \mathrm{m})$ & - & 13942 \\
\hline Resonant Frequency $(\mathrm{MHz})$ & 5.849 & 5.849 \\
\hline Quality Factor, $\mathrm{Q}$ & 1268 & 1268 \\
\hline $\mathrm{S}_{21}(\mathrm{~dB})$ (for tests in the Figure 5, $\left.\mathrm{V}_{\mathrm{p}}=30 \mathrm{~V}\right)$ & -31.8 & -31.8 \\
\hline Input Power, $\mathrm{P}_{\text {in }}(\mathrm{dBm})$ & 10 & 10 \\
\hline On-Chip Amp. Transimpedance, $\mathrm{Z}(\mathrm{M} \Omega)$ & - & 40.6 \\
\hline 20.6 & & \\
\hline
\end{tabular}

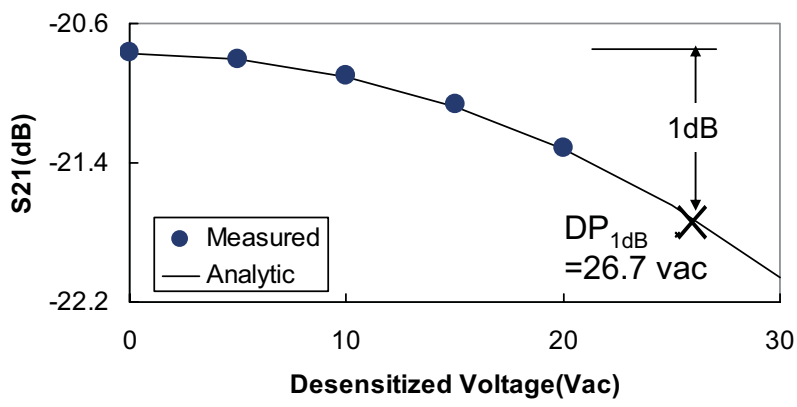

Figure 7. Measured and analytic $S_{21}$ versus the desensitizing voltage, $V_{d}$. The measured data is extracted from Figure 6 and the features applied in analytic model are listed in the Table 1. 


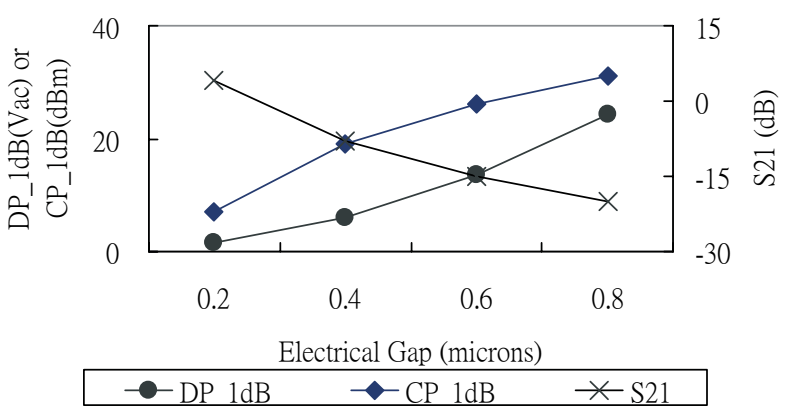

Figure 8. Analytic results of $D P_{1 d B}, C P_{1 d B}$ and $S_{21}$ versus the electrode gap with the assumption that all the features listed in the Table 1 are constant when adjusting the electrode gap.

different frequencies of the desensitizing tone and the magnitude difference between the fundamental tone, $f_{i}$, and the mixing tone, $\left(f_{i}-f_{d}\right)$, are shown in Figure 9. Since the magnitude of mixing tone is $12.5 \mathrm{~dB}$ smaller than the fundamental tone, the nonlinear effect of the amplifiers and network analyzer is insignificant.

Electrical breakdown on the tested device is the major limitation in this device. In one case, a combination of the $V_{d}$ of $30 \mathrm{Vac}$ at the $f_{d}$ of $1 \mathrm{kHz}$ with the $V_{p}$ of $42.5 \mathrm{~V}$ has caused electrical breakdown of an input electrode on the microresonators. However, with improved layout to enhance the isolation of electrical wires, this range might be increased.

Another noticeable phenomenon is the magnitude drop with different $f_{d}$ in the Figure 9, which results from the mechanical resonant shape. Even though this drop is not significant, it causes inaccuracy in the nonlinear models of (7) and the expression of $\mathrm{DP}_{1 \mathrm{~dB}}$ of (8). A complete model is under development.

\section{CONCLUSIONS}

A new desensitization method of characterizing linearity of MEMS resonator filters is introduced. Verifications are performed on a CMOS-MEMS square-frame resonator, showing that the $\mathrm{DP}_{1 \mathrm{~dB}}$ is $26.7 \mathrm{Vac}$ and $\mathrm{CP}_{1 \mathrm{~dB}}$ is calculated to be $32 \mathrm{dBm}$. This method is able to measure nonlinear effect of highly linear resonator filters, with a measuring range larger than that of a $1 \mathrm{~dB}$ compression test or a two-tone test. An analytical expression of $1 \mathrm{~dB}$ desensitization point was presented and verified, which can be used to balance the trade-off between linearity and signal gain in future resonator designs.

\section{ACKNOWLEDGEMENT}

This research effort was supported by the DARPA/MTO NMASP program under award DAAB07-02-C-K001.The authors would like to thank Suresh Santhanam for the developing the vacuum system and the helping with device processing.

\section{REFERENCES}

[1] Sheng-Shian Li, Yu-Wei Lin, Yuan Xie, Zeying Ren, and Nguyen C.T.-C.," Micromechanical hollow-disk ring resonators", Technical Digest of 17th IEEE International Conference on MEMS, Maastricht, The Netherlands(2004), pp. $821-824$.
(A)

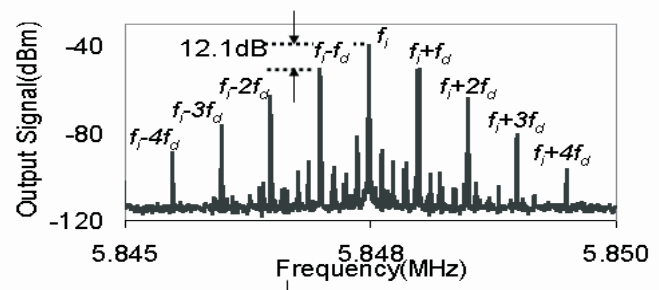

(B)
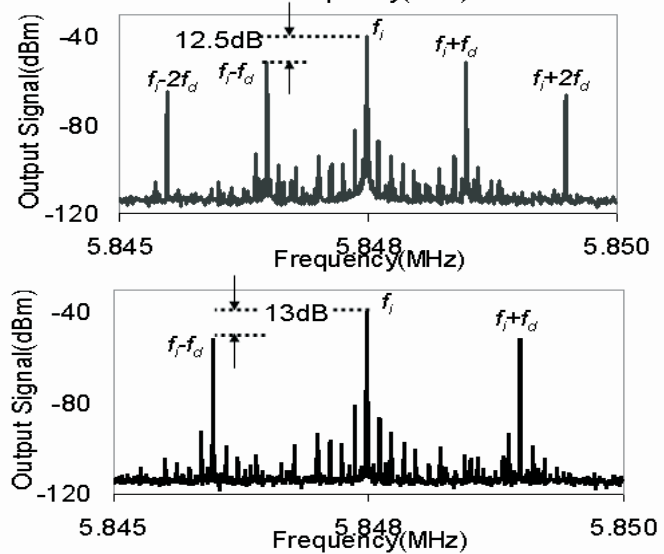

Figure 9. Measured output spectrum of the SFR with input frequency of $5.8474 \mathrm{MHz}$ along with desensitized tone of $15 \mathrm{Vac}$ at different frequency, $f_{p}$. (A) $0.5 \mathrm{kHz}$ (B) $1 \mathrm{kHz}$ and (C) $1.5 \mathrm{kHz}$

[2] Ark-Chew Wong, and Nguyen C.T.-C., "Micromechanical mixer-filters ("mixlers")" Journal of Microelectromechanical Systems, V. 13, 2004, pp. $100-112$.

[3] Liwei Lin, Howe, R.T., and Pisano, A.P., "Microelectromechanical filters for signal processing", Journal of Microelectromechanical Systems, V. 7, 1998 pp. 286 - 294.

[4] Galayko, D., Kaiser, A., Buchaillot, L., Collard, D., and Combi, C., "Electrostatic coupling-spring for micromechanical filtering applications," Proceedings of the 2003 International Symposium on Circuits and Systems, Bangkok, Thailand (2003), pp. 530-533.

[5] Jacques C. Rudell, Jeffrey A. Weldon, Jia-Jiunn Ou, Li Lin, and Paul Gray, "An Integrated GSM/DECT Receiver: Design Specifications," UCB Electronics Research Laboratory Memorandum

[6] B. Razavi, RF Microelectronics, Prentice Hall, 1998

[7] Alastalo, A.T., and Kaajakari, V., "Intermodulation in Capacitively Coupled Microelectromechanical Filters," IEEE Electron Device Letters, VOL. 26, No.5, May 2005, pp. $289-291$

[8] Navid, R.; Clark, J.R.; Demirci, M.; Nguyen, C.T.-C.; "Third-order intermodulation distortion in capacitivelydriven CC-beam micromechanical resonators," Technical Digest of 14th IEEE International Conference on MEMS, Interlaken, Switzerland (2001), , pp. 228-231.

[9] Chiung-Cheng Lo; Fang Chen; Fedder, G.K.; "Integrated HF CMOS-MEMS Square-Frame Resonators with On-Chip Electronics and Electrothermal Narrow Gap Mechanism," Digest of Technical Papers of The 13th International Conference on Solid-State Sensors, Actuators and Microsystems, Seoul, Korea(2005), pp. 2074-2077.

[10] Leonard Meirovitch, Methods of Analytical Dynamics, McGraw-Hill, 1970. 